\title{
The inner fluctuations of the brain in presymptomatic Frontotemporal Dementia: The chronnectome fingerprint
}

Enrico Premi $^{\mathrm{a}, \mathrm{b}}$, Vince D. Calhoun ${ }^{\mathrm{c}, \mathrm{d}}$, Matteo Diano ${ }^{\mathrm{e}, \mathrm{f}}$, Stefano Gazzina ${ }^{\mathrm{a}}$, Maura Cosseddu ${ }^{\mathrm{a}}$, Antonella Alberici $^{\mathrm{a}}$, Silvana Archetti ${ }^{\mathrm{g}}$, Donata Paternicò ${ }^{\mathrm{a}}$, Roberto Gasparotti ${ }^{\mathrm{h}}$, John van Swieten ${ }^{\mathrm{i}}$, Daniela Galimberti ${ }^{\mathrm{j}}$, Raquel Sanchez-Valle ${ }^{\mathrm{k}}$, Robert Laforce Jr. ${ }^{1}$, Fermin Moreno $^{\mathrm{m}}$, Matthis Synofzik ${ }^{\mathrm{n}}$, Caroline Graff $^{\circ}$, Mario Masellis $^{\mathrm{p}}$, Maria Carmela Tartaglia ${ }^{q}$, James Rowe ${ }^{r}$, Rik Vandenberghe ${ }^{s}$, Elizabeth Finger ${ }^{t}$, Fabrizio Tagliavini ${ }^{\mathrm{u}}$, Alexandre de Mendonça ${ }^{\mathrm{v}}$, Isabel Santana ${ }^{\mathrm{w}}$, Chris Butler ${ }^{\mathrm{x}}$, Simon Ducharme ${ }^{\mathrm{y}}$, Alex Gerhard ${ }^{\mathrm{z}}$, Adrian Danek ${ }^{\mathrm{aa}}$, Johannes Levin ${ }^{\mathrm{aa}}$, Markus Otto ${ }^{\mathrm{ab}}$, Giovanni Frisoni $^{\text {ac, ad }}$, Stefano Cappa ${ }^{\text {ac }}$, Sandro Sorbi ${ }^{\text {ae, af }}$, Alessandro Padovani ${ }^{\text {a }}$, Jonathan D. Rohrer ${ }^{\mathrm{ag}}$, Barbara Borroni ${ }^{\mathrm{a} \text {, }}$, on behalf of the Genetic FTD Initiative, GENFI

${ }^{\text {a }}$ Centre for Neurodegenerative Disorders, Neurology Unit, Department of Clinical and Experimental Sciences, University of Brescia, Brescia, Italy

${ }^{\mathrm{b}}$ Stroke Unit, Azienda Socio Sanitaria Territoriale Spedali Civili, Spedali Civili Hospital, Brescia, Italy

${ }^{\mathrm{c}}$ The Mind Research Network, Albuquerque, USA

${ }^{\mathrm{d}}$ Department of Electrical and Computer Engineering, University of New Mexico, Albuquerque, USA

e Department of Psychology, University of Turin, Turin, Italy

${ }^{\mathrm{f}}$ Department of Medical and Clinical Psychology, CoRPS - Center of Research on Psychology in Somatic Diseases, Tilburg University, the Netherlands

${ }^{g}$ Biotechnology Laboratory, Department of Diagnostic, Spedali Civili Hospital, Brescia, Italy

${ }^{\mathrm{h}}$ Neuroradiology Unit, University of Brescia, Italy

${ }^{i}$ Department of Neurology, Erasmus Medical Center, Rotterdam, the Netherlands

j Department of Pathophysiology and Transplantation, "Dino Ferrari" Center, University of Milan, Fondazione Cà Granda, IRCCS Ospedale Maggiore Policlinico, Milan, Italy

${ }^{\mathrm{k}}$ Neurology Department, Hospital Clinic, Institut d'Investigacions Biomèdiques, Barcelona, Spain

${ }^{1}$ Clinique Interdisciplinaire de Mémoire, Département des Sciences Neurologiques, CHU de Québec, Faculté de Médecine, Université Laval, QC, Canada

m Department of Neurology, Hospital Universitario Donostia, San Sebastian, Gipuzkoa, Spain

${ }^{\mathrm{n}}$ Department of Cognitive Neurology, Center for Neurology, Hertie-Institute for Clinical Brain Research, Tübingen, Germany

${ }^{\circ}$ Karolinska Institutet, Department NVS, Center for Alzheimer Research, Division of Neurogenetics, Sweden

${ }^{\mathrm{p}}$ LC Campbell Cognitive Neurology Research Unit, Sunnybrook Research Institute, Toronto, ON, Canada

${ }^{\mathrm{q}}$ Toronto Western Hospital, Tanz Centre for Research in Neurodegenerative Disease, Toronto, ON, Canada

${ }^{\mathrm{r}}$ Department of Clinical Neurosciences, University of Cambridge, Cambridge, UK

${ }^{\mathrm{s}}$ Laboratory for Cognitive Neurology, Department of Neurosciences, KU Leuven, Leuven, Belgium

${ }^{\mathrm{t}}$ Department of Clinical Neurological Sciences, University of Western Ontario, London, ON, Canada

${ }^{\mathrm{u}}$ Fondazione Istituto di Ricovero e Cura a Carattere Scientifico Istituto Neurologico Carlo Besta, Milan, Italy

${ }^{\mathrm{v}}$ Faculty of Medicine, University of Lisbon, Lisbon, Portugal

${ }^{\mathrm{w}}$ Neurology Department, Centro Hospitalar e Universitário de Coimbra, Portugal

${ }^{x}$ Department of Clinical Neurology, University of Oxford, Oxford, UK

${ }^{\mathrm{y}}$ Department of Neurology and Neurosurgery, McGill University, Montreal, Quebec, Canada

${ }^{\mathrm{z}}$ Institute of Brain, Behaviour and Mental Health, The University of Manchester, Withington, Manchester, UK

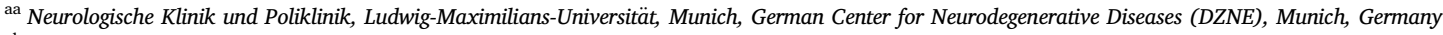

${ }^{\mathrm{ab}}$ Department of Neurology, University Hospital Ulm, Ulm, Germany

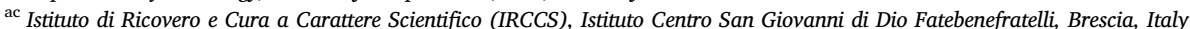

ad Memory Clinic and LANVIE-Laboratory of Neuroimaging of Aging, University Hospitals and University of Geneva, Geneva, Switzerland

${ }^{\text {ae }}$ Department of Neuroscience, Psychology, Drug Research and Child Health, University of Florence, Florence, Italy

af Istituto di Ricovero e Cura a Carattere Scientifico (IRCCS) "Don Gnocchi", Florence, Italy

${ }^{\text {ag }}$ Dementia Research Centre, UCL Institute of Neurology, UK

\footnotetext{
* Corresponding author. Neurology Unit, University of Brescia, Piazza Spedali Civili 1, Brescia, 25125, Italy.

E-mail address: bborroni@inwind.it (B. Borroni).
} 


\section{A R T I C L E I N F O}

\section{Keywords:}

Frontotemporal dementia

Mutation

Granulin

Microtuble associate protein tau

C9orf72

resting-state fMRI

Dynamic brain functional connectivity

Chronnectome

\section{A B S T R A C T}

Frontotemporal Dementia (FTD) is preceded by a long period of subtle brain changes, occurring in the absence of overt cognitive symptoms, that need to be still fully characterized. Dynamic network analysis based on restingstate magnetic resonance imaging (rs-fMRI) is a potentially powerful tool for the study of preclinical FTD.

In the present study, we employed a "chronnectome" approach (recurring, time-varying patterns of connectivity) to evaluate measures of dynamic connectivity in 472 at-risk FTD subjects from the Genetic Frontotemporal dementia research Initiative (GENFI) cohort.

We considered 249 subjects with FTD-related pathogenetic mutations and 223 mutation non-carriers (HC). Dynamic connectivity was evaluated using independent component analysis and sliding-time window correlation to rs-fMRI data, and meta-state measures of global brain flexibility were extracted.

Results show that presymptomatic FTD exhibits diminished dynamic fluidity, visiting less meta-states, shifting less often across them, and travelling through a narrowed meta-state distance, as compared to HC. Dynamic connectivity changes characterize preclinical FTD, arguing for the desynchronization of the inner fluctuations of the brain. These changes antedate clinical symptoms, and might represent an early signature of FTD to be used as a biomarker in clinical trials.

\section{Introduction}

Resting state functional magnetic resonance imaging (rs-fMRI) has become a useful tool to investigate the connectivity changes in neurodegenerative dementias (Pievani et al., 2014a; Premi et al., 2014b). Spontaneous brain activity at rest is organized in functionally specialized large-scale networks, that roughly correspond to different functional domains and that are selectively damaged by various neurodegenerative conditions (de Pasquale et al., 2017).

However, previous results make the implicit assumption that the functional coupling among brain regions is static and unchanging over short periods of time (Damoiseaux et al., 2006; De Luca et al., 2006; Liu et al., 2018).

This concept has since been modified with analytic approaches that capture the fact that the human brain is an interacting dynamic network and its architecture of coupling among brain regions varies across time (termed "the chronnectome") (Calhoun et al., 2014; Canolty et al., 2010; Chang and Glover, 2010; Fries, 2005; Hillebrand et al., 2016; Sakoglu et al., 2010). Dynamic connectivity studies have demonstrated reoccurring patterns of brain functional connectivity, or functional connectivity "states", that are reproducible over time and across subjects (Allen et al., 2014; Chang and Glover, 2010; Marusak et al., 2017). Initial dynamic connectivity studies were based on the assumption that subjects were allowed to be in only one "state" at a given point in time, while recent work has introduced the concept of "meta-states", suggesting that subjects may be in multiple states to varying degrees at the same point in time (Calhoun et al., 2014; Miller et al., 2016). Thus, for instance, if in the time-course we are able to identify six distinct states of functional dynamic connectivity, at a given point in time each subject will have a weighted probability to be in more than one state (Miller et al., 2016). Meaningful measures of meta-state dynamic fluidity, such as the number of meta-states a subject passes through or the number of switches from one meta-state to another, have been suggested as an intuitive way to characterize global dynamic connectivity behaviour, showing promise for predicting mental states and cognitive performances (Liu et al., 2018; Preti et al., 2017). From this point of view, meta-state measures could provide a more "global" information on the effect of an ongoing neurodegenerative process, overcoming the evaluation of single specific brain areas or connectivity pathways, and evaluating global perturbation of the brain activity's temporal dynamics (Calhoun et al., 2014; Miller et al., 2016).

Frontotemporal Dementia (FTD) is a neurodegenerative disease characterized by behavioural abnormalities, impairment of executive functions and language deficits (Gorno-Tempini et al., 2011; Rascovsky et al., 2011) and defined by focal frontotemporal atrophy (Whitwell et al., 2009). In a significant proportion of the cases, FTD is an inherited autosomal dominant disorder; mutations in the Granulin (GRN), chromosome 9 open reading frame 72 (C9orf72) or Microtuble Associated Protein Tau (MAPT) genes drive up to $\sim 40 \%$ of Mendelian cases (Borroni et al., 2008b). In genetic FTD, the neural substrates associated with the presymptomatic stage need to be fully characterized: although the perturbation of static large-scale networks has already been demonstrated (Dopper et al., 2014; Lee et al., 2017; Premi et al., 2014a; Whitwell et al., 2011; Zhou et al., 2010), the specific findings were not fully consistent, in particular for Salience network. In fact, both an increased connectivity in medial frontal regions in GRN carriers (Borroni et al., 2008a; Premi et al., 2014b) (specially within the Salience Network), and a reduced seed-based connectivity between anterior cingulate cortex and posterior regions of the Default Mode Network in a group of GRN and MAPT carriers were reported (Dopper et al., 2014). Furthermore, a recent study on presymptomatic C9orf72 carriers showed a reduced functional connectivity in all the studied networks (Salience, Default Mode, Sensorimotor and medial pulvinar networks) (Lee et al., 2017). Finally, a study on a small group of MAPT carriers demonstrated an altered functional connectivity in the Default Mode Network with no alteration of Salience Network (Whitwell et al., 2011). The evaluation of large-scale structural network topology along with their temporal dynamics might offer a theoretical framework that can contribute to understand the earliest abnormalities in FTD, with the new perspective of whole brain assessment (Avena-Koenigsberger et al., 2017). Thus, by a data-driven approach, we did not study single specific brain pathways, but the global dynamic perturbation of the brain in the presymptomatic phase of FTD.

These premises set the stage for the present study, in which we analyzed dynamic brain connectivity in presymptomatic subjects carrying GRN, MAPT or C9orf72 mutations with the purpose $a$ ) to assess the chronnectome fingerprint by considering meta-state measures; $b$ ) to study the association between chronnectome changes and cognitive performances; and c) to correlate chronnectome changes with expected age at disease onset, to evaluate if meta-state measures are associated with proximity to clinical onset. To this end, we analyzed rs-fMRI data of 472 subjects from the Genetic Frontotemporal Dementia Initiative (GENFI) cohort (http://genfi.org.uk) using a dynamic functional network connectivity (dFNC) approach to investigate the chronnectome in presymptomatic mutations carriers as compared to mutation non-carriers.

\section{Methods}

\subsection{Subjects}

Data for this study were drawn from the GENFI multicenter cohort study, which consists of 23 research centers in Europe and Canada. 
Table 1

Demographic characteristics of included participants.

\begin{tabular}{llll}
\hline Characteristic & $\begin{array}{l}\text { Carriers } \\
(\mathrm{n}=249)\end{array}$ & $\begin{array}{l}\text { Non-carriers } \\
(\mathrm{n}=223)\end{array}$ & $\begin{array}{l}\text { P- } \\
\text { value }^{\mathrm{a}}\end{array}$ \\
\hline Age (years) & $44.7 \pm 11.7$ & $47.2 \pm 13.2$ & 0.042 \\
Female, \% & $64.7 \%$ & $56.5 \%$ & 0.073 \\
Education (years) & $14.4 \pm 3.2$ & $14.1 \pm 3.3$ & 0.242 \\
Years at expected onset & $-13.8 \pm 11.3$ & - & - \\
$\quad$ (years) & & & \\
Cognitive and behavioural assessment & $29.2 \pm 1.1$ & $29.4 \pm 0.9$ & 0.633 \\
MMSE & $4.45 \pm 8.2$ & $3.3 \pm 6.1$ & 0.098 \\
CBI-R & $-2.5 \pm 64.5$ & $-10.2 \pm 70.3$ & 0.075 \\
TMT-A (Z-scores) & $-8.2 \pm 80.7$ & $-14.4 \pm 69.0$ & 0.345 \\
TMT-B (Z-scores) & &
\end{tabular}

a Mann-Whitney $U$ test, otherwise specified; Chi-Square test; results are expressed as mean \pm standard deviation, otherwise specified. MMSE: MiniMental State Examination; CBI-R: Cambridge Behavioural Inventory Revised version; TMT-A: part A of the Trial Making Test; TMT-B: part B of the Trial Making Test.

Inclusion and exclusion criteria have been previously described (Rohrer et al., 2015). Local ethics committees approved the study at each site and all participants provided written informed consent according to the Declaration of Helsinki.

We considered asymptomatic participants at risk to carry GRN, C9orf72 or MAPT mutations. Between January 2012 and January 2017, we considered 472 participants, of which 249 were mutation carriers (45 with MAPT, 122 with GRN, and 82 with C9orf72 mutations) and 223 were mutation non-carriers. Subjects were enrolled from 18 centers belonging to the GENFI network (4 centers were excluded from the present project for image artifacts, very low number of included subjects $(<2)$, or for using 1.5T MRI scanner); the MRI parameters for each of the 18 included centers was reported in Supplementary Table 1. Demographic characteristics of mutation carriers and mutation non-carriers are reported in Table 1.

Estimated years from expected symptom onset in presymptomatic mutation carriers were calculated as the age of the participant at the time of the study assessment minus the mean familial age at symptom onset, as previously reported (Rohrer et al., 2015).

Included at-risk subjects underwent a careful recording of demographic data and a standardized clinical and neuropsychological assessment (derived from the Uniform Data Set (Morris et al., 2006)), as previously published (Premi et al., 2017). We considered tests highly sensitive to identify initial changes in presymptomatic genetic FTD, as previously reported (Rohrer et al., 2015). Thus, we considered assessment of behavioural symptoms with the Cambridge Behavioural Inventory Revised version (CBI-R) (Wear et al., 2008), general cognitive function with the Mini-Mental State Examination (MMSE) (Morris et al., 2006), and cognitive processing speed and executive functions assessed with the part A and part B of the Trial Making Test (TMT) (Morris et al., 2006), respectively. For each test, apart from the MMSE and CBI-R, we calculated Z scores based on language-specific norms (Rohrer et al., 2015). Neuropsychological evaluation was harmonized across sites.

\subsection{MRI acquisition}

MRI protocol was common to all the GENFI sites, and adapted for different scanners; no pre-study phantom harmonization was performed at local level. In summary, T2-weighted echo planar imaging (EPI) sequences sensitized to blood oxygenation level dependent (BOLD) contrast for rs-fMRI were considered in the present study (see Supplementary Table 1 for details on the fMRI protocol used by each site). As the repetition times (TRs, ranging from $2200 \mathrm{~ms}$ to $2500 \mathrm{~ms}$ ) and the volume numbers (ranging from 140 to 200) varied across the GENFI centers, we considered only the first 140 vol of the EPI images for each subject (mean acquisition time: $311.5 \pm 4.95 \mathrm{~s}$ ). During scanning, subjects were asked to keep their eyes closed, not to think of anything in particular, and not to fall asleep.

\subsection{Neuroimaging pre-processing and analysis}

Functional data were pre-processed using the toolbox for Data Processing \& Analysis for Brain Imaging (DPABI, http://rfmri.org/dpabi) (Yan et al., 2016) based on the Statistical Parametric Mapping (SPM12) software.

For each subject, the first 2 volumes of the fMRI series were discharged to account for magnetization equilibration. The remaining 138 volumes underwent slice-timing correction and were realigned to the first volume. Any subject who had a maximum displacement in any direction larger than $2.5 \mathrm{~mm}$, or a maximum rotation $(\mathrm{x}, \mathrm{y}, \mathrm{z})$ larger than $2.5^{\circ}$, was excluded. We considered absolute (mean translation and mean rotation) and relative (framewise displacement (FD): Power (FD-P) (Power et al., 2012), Jenkinson (FD-J) (Jenkinson et al., 2012), Van Dijk (FD-VD) (Van Dijk et al., 2012)) and DVARS (D for the temporal derivative of time courses, VARS referring to RMS, root mean squared head position change) (Power et al., 2012) (see Supplementary Table 2) motion parameters. Data were subsequently spatially normalized to the EPI unified segmentation template in Montreal Neurological Institute coordinates derived from SPM12 software and resampled to $3 \times 3 \times 3$ cubic voxels. We preferred a normalization to the EPI template (instead of T1-based normalization) in line with recent data demonstrating that EPI normalization is able to reduce variability across subjects (especially when EPI distortion correction is not applied, as in our case) and boost the effective sample size by 15-25\%. Furthermore, studies assessing distance maps and intra subject variability in multicentre cohorts by EPI normalization are comparable to our data (Calhoun et al., 2017). Spatial smoothing with an isotropic Gaussian kernel with full-width at half-maximum (FWHM), $10 \mathrm{~mm}$ was applied; this threshold smoothing value was chosen for a number of reasons: 1) we assessed dFC within large areas, which are not usually affected by a relative large spatial smoothing; 2) we adopted Abrol's template to estimate the dFC (Abrol et al., 2017) (which has been calculated on 7500 healthy subjects), and consequently we opted for similar fMRI pipeline (Abrol et al., 2016) $(10 \mathrm{~mm}$ FWHM); 3) spatial smoothing of $8-10 \mathrm{~mm}$ FWHM is recommended to increase sensitivity (Mikl et al., 2008).

\subsection{Functional networks decomposition}

The functional imaging data were processed using the GIFT (GIFT toolbox, http://mialab.mrn.org/software/gift) (Calhoun et al., 2001) and a spatially constrained ICA algorithm (Wang et al., 2016) called Group Information Guided independent component analysis (GIG-ICA) was used to compute spatial maps that corresponded to those from a previous analysis (Du et al., 2016). In this approach, brain network spatial maps are used as reference templates to calculate functional networks for each individual subject one-by-one by maximizing independence in the context of the spatial constraint. These template maps include the brain networks with a neuronal origin (not artefactual) and assign the remaining data to be noise. We take advantage from the recently published set of 37 spatial maps derived from 7500 healthy subjects as spatial references for our network selection (Abrol et al., 2017). We then considered only cortical and subcortical networks, and we discharged cerebellar networks due to incomplete coverage of cerebellum in our sample, thus considering 35 spatial maps. The TR of each subject was entered in GIFT pre-processing, and we accounted for the differences in EPI acquisition protocols among centers. The Infomax approach was applied (Bell and Sejnowski, 1995) to estimate the independent group components and 35 functional networks were considered 
(see Supplementary Fig. 1 for details). Subject-specific spatial patterns and time-courses were derived using spatial-temporal regression and then converted to Z-scores. The single time courses were detrended (to remove baseline drifts from the scanners and/or physiological pulsations), orthogonalized with respect to 12-motion parameters, despiked (replacement of outlier time points with 3rd order spline fitting to clean neighbouring points) and filtered using a 5th order Butterworth filter (0.01-0.15 Hz) (Abrol et al., 2016).

\subsection{Windowed functional network connectivity and correlation patterns decomposition (meta-states)}

The dynamic functional network connectivity (dFNC) was achieved using dynamic FNC toolbox implemented in GIFT (Damaraju et al., 2014). dFNC was assessed using a sliding-window approach to estimate correlation matrices between components for each segment. Segments were defined with a tapered window convolving a rectangle (width $=30$, TRs $=66 \mathrm{~s}$ ) with a Gaussian $(\sigma=3)$ and slide in steps of 1 TR. A LASSO approach with L1 regularization (100 repetitions) was used to compute the covariance between the independent component (IC) time-courses. To obtain the decomposition into connectivity patterns (CPs), the spatial ICA (sICA) approach was applied, considering a number of CPs of 6 , in line with previous work on metastates in dynamic brain connectivity (5-6 CPs used) (Allen et al., 2014; Miller et al., 2016). As previously described, the time-courses were discretized (to work over a more tractable space) into 8 bins (positive and negative quartiles) and each timepoint was ended into a meta-state (Miller et al., 2014). The time-courses for sICA CPs were derived from the regression of each subject's dFNC information at each time window on the group of sICA CPs. During dFNC preprocessing the following covariates of no interest were considered: age, gender, acquisition site, scanner type, family number, genetic status of the proband, DVARS index (Power et al., 2012) and the variance associated with them has been regressed out from the windowed dynamic functional network connectivity correlations for each subject at this processing step. Furthermore, the potential confounding effect of motion (DVARS index) across groups and the correlation with metastate measures were explored.

Four indexes of connectivity dynamism were considered: $i)$ the number of distinct meta-states the subjects occupied during their scans (meta-state number); ii) the number of times that subjects switch from one meta-state to another (meta-state changes), iii) the largest distance of two meta-states that subjects occupied (meta-state span), and $i v$ ) the overall distance travelled by each subject through the state space (the sum of the L1 distances between successive meta-states, i.e. meta-state total distance). Metastate indexes are global (not state specific) measures the describe the trajectory of the windowed correlations among the different states. In meta-state framework, subject's state can be represented by varying degrees of multiple states, with lesser distortion in the $\mathrm{CPs}$, considering that contributions of all overlapping states were recognized.

\subsection{Statistical analysis}

The assumption of normality for continuous variables was not satisfied for all group combinations, as assessed by Shapiro-Wilk's test $(\mathrm{p}<0.05)$. Thus, comparisons of demographic and clinical characteristics between groups (mutation carriers vs. mutation non-carriers) were assessed by Mann-Whitney $U$ test for continuous variables and $\chi^{2}$ test for categorical variables. Pearson's correlation was used to assess the relationship between the meta-state measures (meta-state number, metastate changes, meta-state span and meta-state total distance) and age at expected symptom onset. Finally, partial correlation (considering age as a nuisance variable) was used to test the relationship between meta-state measures and cognitive/behavioural performances (CBI-R, MMSE, TMTA, TMT-B). All the statistical analysis was performed using IBM SPSS
Statistics 22.0 (Chicago, USA) and statistical significance level set at $\mathrm{p}<0.05$, corrected for multiple comparisons (Benjamini-Hochberg FalseDiscovery-Rate (FDR) correction (Benjamini and Hochberg, 1995)), considering four meta-state measures and four clinical tests. Direct comparisons (mutations carriers vs mutation non-carriers) and correlation analyses (between age at expected symptom onset and meta-state measures and between clinical tests and meta-states measures) were carried out. Taking into account that preprocessing (Independent Component Analysis, ICA) was performed using all subjects together and it was not completely optimized for the subgroup analysis (requiring separated preprocessing for each mutation group but making the evaluation of the main effect hardly interpretable) we performed exploratory analyses considering each gene (GRN, C9orf72 or MAPT) separately versus mutation non carriers.

\subsection{Data and code availability statement}

The data used to support the findings of this study were derived from the Genetic Frontotemporal Dementia Initiative (GENFI, http://genfi. org.uk/). They are available on request from the Principal Investigator of the GENFI consortium (Dr Jonathan Rohrer, University College London, genfi@ucl.ac.uk).

\section{Results}

Two hundred-forty nine mutation carriers (82 with C9orf72, 122 with GRN and 45 with MAPT mutations) were considered, and compared with 223 mutation non-carriers. Considering clinical and demographic variables, mutation non-carriers were slightly older than mutation carriers $(47.2 \pm 13.2$ vs $44.7 \pm 11.7, \mathrm{p}=0.042)$ (see Supplementary Table 2 for details). We considered six connectivity patterns (CPs) of dFNC, which are reported in Fig. 1. The colors of each CP represent the direction and the strength of the correlation among the 35 considered network components (red: positive correlation and blue: negative correlation).

dFNC was expressed as a weighted sum of the discretized sixdimensional CPs, for each given point in time and for each subject. Mutation carriers exhibited diminished dynamic fluidity, as they occupied a fewer number of meta-states (i.e., meta-state numbers) and changed from one meta-state to another less often (i.e., meta-state changes) than mutation non-carriers (see Table 2). Furthermore, mutation carriers operated over a restricted dynamic range with decreased meta-state total distance, as they travelled less overall distance, between successive meta-states, through the state space than mutation noncarriers (see Table 2). We did not find any difference in meta-state span between groups.

Taking into account the statistically significant difference of age between groups (mutation non-carriers were older then mutation carriers) we also performed an exploratory analysis considering a subgroup of non-carriers $(n=200)$ with a comparable age versus mutation carriers. As reported in Supplementary Analysis 1, the results were similar to the original groups, with a significantly altered meta-state dynamic connectivity in mutation-carriers, supporting the idea that age at visit did not explain meta-state differences.

The potential confounding effect of motion on meta-state measures has been tested (see Supplementary Table 2 and Supplementary Table 3): no significant differences in DVARS values among groups (C9orf72, GRN, $M A P T$ versus mutation-negative carriers) as well as no significant correlations between DVARS values and meta-state measures were evident. When other parameters of motion were considered no significant differences between groups (also considering the three mutations separately) were found. Furthermore, a weak (even if statistically significant) correlation between motion parameters (i.e. FD) and meta-state measures was evident (see Supplementary Table 3). However, with a selection of subjects (either mutation carriers and non-carriers) with lowmovement (mean $\mathrm{FD} \leq 0.2 \mathrm{~mm}$ in line with literature data) (Parkes 


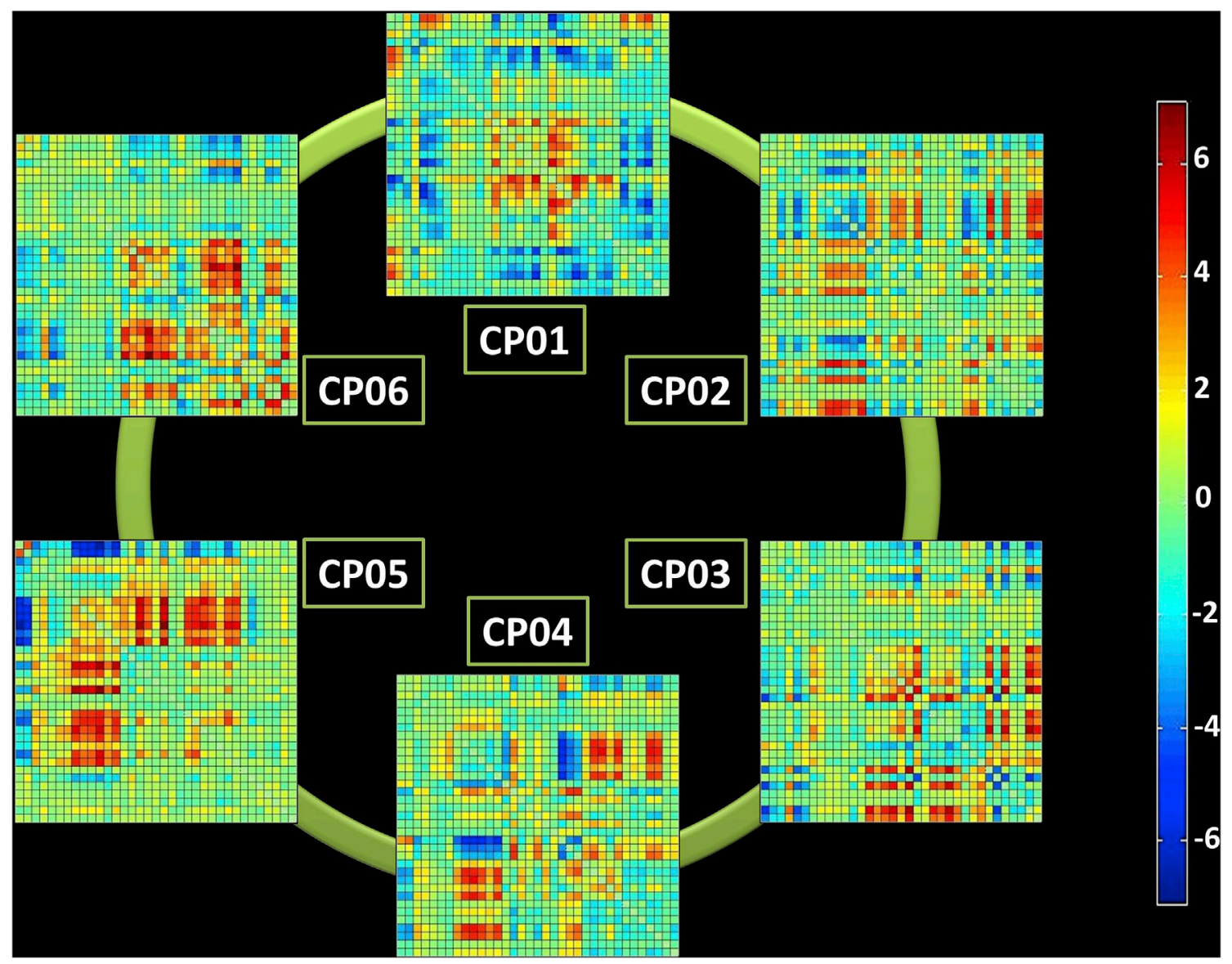

Fig. 1. The six connectivity patterns (CPs) resulting from the dynamic Functional Network Connectivity (dFNC) analysis. The six correlations' matrix (among the 35 considered network components) are reported. The colorbar represents the direction and the strength of each correlation (red: positive correlation, blue: negative correlation). 
Table 2

Meta-state measures in the studied groups.

\begin{tabular}{llll}
\hline Variable & $\begin{array}{l}\text { Carriers } \\
(\mathrm{n}=249)\end{array}$ & $\begin{array}{l}\text { Non-carriers } \\
(\mathrm{n}=223)\end{array}$ & $\mathrm{p}^{\mathrm{a}}$ \\
\hline $\begin{array}{c}\text { Number of distinct metastates, } \\
\text { mean } \pm \text { SD }\end{array}$ & $51.0 \pm 8.9$ & $53.1 \pm 8.8$ & 0.024 \\
$\begin{array}{c}\text { Number of meta-state changes, } \\
\text { mean } \pm \text { SD }\end{array}$ & $51.3 \pm 8.5$ & $53.3 \pm 8.1$ & 0.024 \\
$\begin{array}{l}\text { Meta-state span, mean } \pm \text { SD } \\
\begin{array}{c}\text { Meta-state total distance, } \\
\text { mean } \pm \text { SD }\end{array}\end{array}$ & $23.0 \pm 4.7$ & $23.8 \pm 4.5$ & 0.136 \\
\hline
\end{tabular}

a Mann-Whitney $U$ test (carriers vs non-carriers) FDR-corrected for multiple comparisons; SD: standard deviation.

et al., 2018), similar statistically significant findings on meta-states indexes (mutation-carriers versus mutation non-carriers) were demonstrated (see Supplementary Analysis 2).

In Fig. 2, meta-state dynamics through time, meta-state numbers, meta-state change points, and meta-state total distance in a representative mutation carrier and in a representative mutation non-carrier were reported. Representative mutation non-carrier showed a greater brain dynamism, as compared to a representative mutation carrier (panel A), as suggested by the more complex pattern in the former subject, with an higher number of realized meta-states (panel B), meta-state changes (panel C), and greater travelled overall distance (panel D), compared to the mutation carrier subject.

In the exploratory analysis (not corrected for multiple comparisons), we evaluated C9orf72, GRN and MAPT mutation carriers separately, as compared to HC. C9orf72 mutation carriers showed reduced meta-states numbers $(\mathrm{p}=0.032)$ and reduced meta-state changes $(\mathrm{p}=0.041) ;$ MAPT mutation carriers had reduced meta-states numbers $(\mathrm{p}=0.042)$ and reduced overall meta-state total distance $(\mathrm{p}=0.046)$, while we did not find significant findings in GRN mutation carriers, as compared to HC.

The correlation between meta-state measures and age at expected onset in mutation carriers was then considered (FDR-corrected for multiple comparisons). The closer the age at expected symptom onset, the lower the number of meta-states (Pearson's correlation, $r=-0.174$, $\mathrm{p}=0.012)$, the lower the meta-state changes $(\mathrm{r}=-0.166, \mathrm{p}=0.012)$, the lower the meta-state span $(r=-0.167, \mathrm{p}=0.012)$ and the lower the meta-state total distance $(\mathrm{r}=-0.141, \mathrm{p}=0.027)$ was found. As exploratory analysis, we also tested the aforementioned correlation in the three groups of mutations (see Supplementary Table 4) demonstrating that the effect was mainly supported by C9orf72 mutation carriers.

Finally, the correlation between meta-state measures and cognitive performance in mutation carriers and mutation non-carriers, considering age at evaluation as a covariate, was assessed (not corrected for multiple comparisons). TMT-A scores (the higher the scores the worse the performances) were inversely correlated with meta-state span $(r=-0.143$, $\mathrm{p}=0.024)$ and meta-state total distance $(\mathrm{r}=-0.124, \mathrm{p}=0.050)$ : interestingly, exploring the correlation of TMT-A scores and meta-state measures, the inverse correlation with meta-state span was primarily related to C9orf72 group ( $\mathrm{r}=-0.281, \mathrm{p}=0.011$, not corrected for multiple comparisons). No other significant correlation between meta-state measures and neuropsychological/behavioural tests in the three mutations, separately, was demonstrated. No other significant correlations between meta-state measures and MMSE, CBI-R or TMT-B were found.

\section{Discussion}

In this study, results showed consistent evidence of reduced global flexibility and dynamism in the brain of presymptomatic FTD, which progressively worse with proximity to age at expected symptoms onset. Moreover, the impairment of global inner fluctuations of the brain was well correlated with processing speed performances in asymptomatic subjects carrying pathogenetic FTD mutations.

In the last decade, rs-fMRI has been used to estimate functional brain connectivity, considering regions with temporally coherent brain activity as "functional brain networks" (Buckner et al., 2009; Jafri et al., 2008). Up to now, most studies have relied on two implicit assumptions: the first is a "spatial assumption", that each brain region participates in exactly one network, and the second is a "temporal assumption", that the connectivity within each network are essentially static over time (Allen et al., 2014; Ciric et al., 2017; Faghiri et al., 2018; Hutchison et al., 2013). New evidence clearly suggests that the brain is dynamically multistable, and spontaneous low-frequency fluctuations in BOLD fMRI data during the acquisition capture reoccurring patterns (states) of interactions among intrinsic networks at rest (chronnectome) (Calhoun et al., 2014; Onton and Makeig, 2006). This is in line with spontaneous activity fluctuations found in electrophysiological studies (Arieli et al., 1996; Pascual-Marqui et al., 1995; Yanagawa and Mogi, 2009). This "dynamic" inter-regional connections showed a high degree of reproducibility (data analysis methods, grouping, decomposition techniques, quality of the data, methodological validation with surrogate data analysis) (Abrol et al., 2016, 2017). Such findings open a new chapter in the study of neurodegenerative diseases, offering a different perspective to investigate the earliest brain changes, thus considering global brain connectivity instead of either single network connectivity or focal neural damage.

In the present study, we assessed the chronnectome fingerprint in preclinical monogenic FTD by considering meta-states. Meta-states properly describe whole brain flexibility, moving from the concept that each subject may be in a defined "state" of functional dynamic connectivity in a given point in time to the concept that a subject may have a weighted probability to be in more "states" in each given point in time. Thus, in each subject, dynamic connectivity was represented by the probability sum of the different connectivity patterns at a given point in time. From this point of view, we explored those indexes (metastate numbers, changes, span, and total distance) related to the global dynamic properties of the brain rather than specific states. This core challenge allows us to better identify early and hidden features of brain disorders, as already demonstrated in schizophrenia (Miller et al., 2016).

Herein, we reported that asymptomatic mutation carriers a) passed through a lower number of distinct meta-states (i.e., lower meta-state number); b) less often switched between meta-states (i.e., lower metastate changes), and c) switched frequently between two meta-states at close distal boundaries of the state space (i.e., lower mate-state total distance), as compared to mutation non-carriers. Altogether, these findings point to a precocious impairment of the inner fluctuations of the brain with an effect on at-distance networks through a diminished dynamic fluidity (meta-state number and meta-state changes) and a restricted dynamic range (meta-state total distance) in preclinical FTD (Warren et al., 2013).

Prior work has primarily focused on topological differences among networks in FTD, identifying structural and even functional changes of specific brain networks in preclinical disease (Caroppo et al., 2015; Dopper et al., 2014; Moreno et al., 2013; Pievani et al., 2014b; Premi et al., 2014a, 2014b, 2016; Rohrer et al., 2015), evaluating neuropathological progression according to the molecular nexopathy paradigm (Warren et al., 2012). In the present work, we suggested that FTD at the earliest disease stages affects whole brain efficiency, providing a complementary view of presymptomatic FTD. From this point of view, the potential perturbation of meta-state measures in the single mutation (as supported by the exploratory analyses) should deserve attention with separated dynamic connectivity analyses in each group.

Moreover, we reported that the greater the meta-state abnormalities in mutation carriers, the closer the age at expected onset, in line with the progressive changes which in turn lead to symptom onset and structural damage in FTD. However, it should be noted that the degree of correlation between meta-state perturbations and expected age at onset was very low and statistically significant only in C9orf72 mutation carriers. Finally, TMT-A, a test reflecting processing speed skills (Bowie 
A

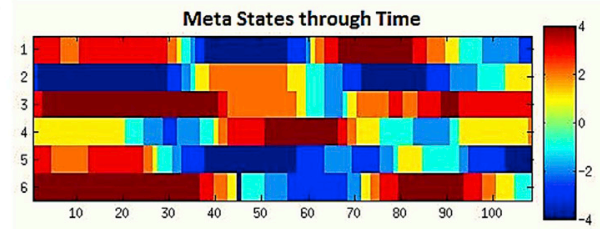

B

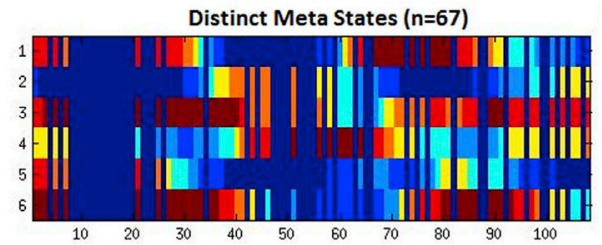

C

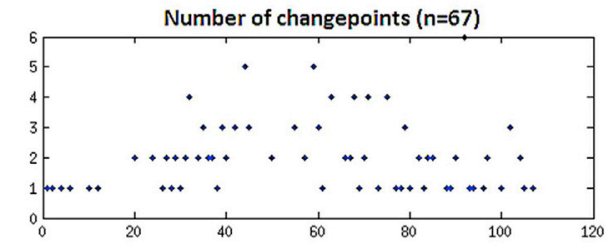

D

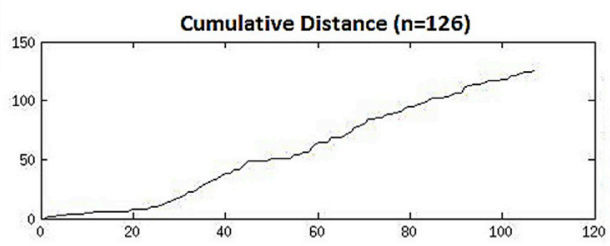

Mutation non-carrier (male, 39 y.o.)

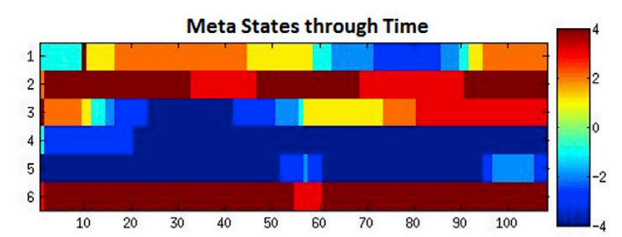

A

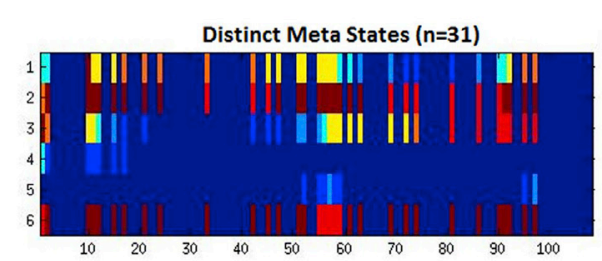

B

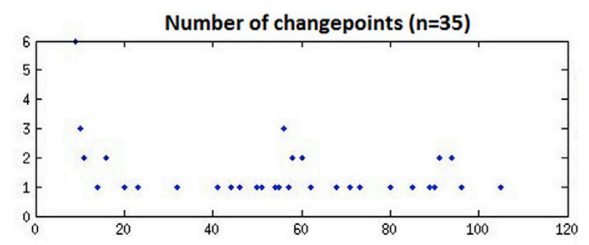

C

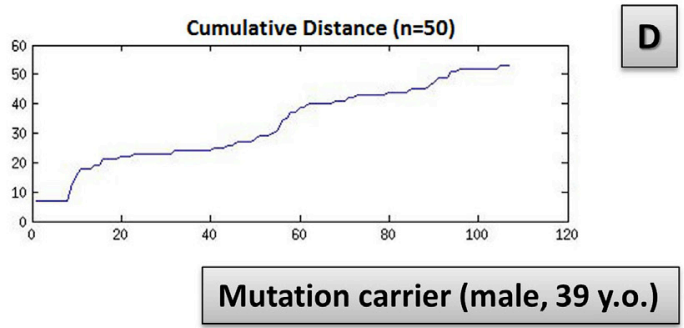

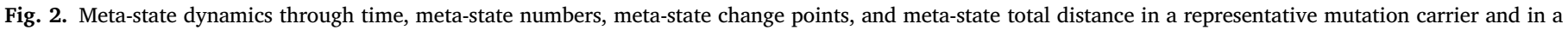
representative mutation non-carrier. 
and Harvey, 2006), inversely correlated with measures of dynamic range connectivity. This confirms and extends the previous hypothesis of a strict link between chronnectome fingerprint and cognition performances (Chen et al., 2016; Jia et al., 2014) that needs to be further explored, also considering the strength of correlations in the mutation-carriers group. The lack of correlation between cognitive/behavioural performances and meta-state measures might be addressed to the weak abnormalities of cognition and behaviour in preclinical FTD (Rohrer et al., 2015). Conversely, TMT-A, an index of preprocessing speed skill, is one of the early cognitive marker in preclinical FTD (Rohrer et al., 2015). The absence of a significant correlation between TMT-A scores and meta-state measures in healthy controls further supports the idea that this finding is not age-driven but mutation-driven. Our study presents a number of limitations that need to be acknowledged. First, the influence of vigilance (even though rs-fMRI data were collected with closed eyes) was not evaluated (Wang et al., 2016). Second, subject motion is of particular concern in dynamic analyses of rs-fMRI (Wang et al., 2016). To overcome this limit, we included motion parameters estimation as well as DVARS index (that describes the rate of change of BOLD signal across the entire brain at each frame of the data compared with the next one; in this sense, DVARS represents a measure of how much the intensity of a brain image changes in comparison to the previous timepoint) in the preprocessing and in the statistical design, respectively (Abrol et al., 2016; Power et al., 2012). Furthermore, also considering further indexes of absolute and relative movement (i.e. framewise displacement, FD) we tested the relationship between the aforementioned indexes and metastate measures as well as with cognitive performances: as described in Supplementary Table 3 a residual significant relationship between relative movement indexes (in particular FD) was evident, supporting the hypothesis of a potential influence of the movement, even after the multi-level correction. However, to further corroborate our findings we performed an exploratory analysis (Supplementary Analysis 3) only considering a selection of carriers and non-carriers with low-movement, with comparable findings. Third, considering the unconstrained nature of the resting-state signal, thought content during the scan represented a significant source of variability, can only be partially evaluated by retrospective questionnaires (Marusak et al., 2017; O'Callaghan et al., 2015). Four, scan time of acquisition was in line with (or even greater than) previous studies (Allen et al., 2014; Marusak et al., 2017) even though the development of effective dynamic functional connectivity statistical approaches is still an open field, deserving attention in the future (Hindriks et al., 2016; Miller et al., 2016; Shakil et al., 2016; Shine et al., 2015; Yaesoubi et al., 2015). In line with this, the utilization of nuisance covariates (in particular in multi-center studies) represents a challenging issue, considering the different types of confounders (continue or categorical variables) as well as the time-point to remove the variance associated with them. As described in the Methods section we performed nuisance variables regression during the windowed functional network connectivity processing before metastate calculation, even if this was not completely standardized for dynamic connectivity analysis and should be considered as limitation. Fifth, the choice of the dimension of CPs decomposition (6 CPs), even if in line with previous studies, is arbitrary: from this point of view, a data-driven approach (elbow criterion of the cluster validity index as for state measures calculation) (Marusak et al., 2017; Rashid et al., 2014, 2016) should be implemented also for metastates analysis to increase the overall standardization. To obtain the decomposition into connectivity patterns (CPs), the spatial ICA (sICA) approach was applied, considering a number of CPs of 6 , in line with previous work on metastates in dynamic brain connectivity (5-6 CPs used).

Despite these limitations, to the best of our knowledge, this is the first study applying chronnectome approach to neurodegenerative dementias. The exploration of time-varying aspects of functional connectivity unveiled aspects of the underappreciated early brain changes in FTD: beside the well-established concept of the selective vulnerability of specific brain regions (molecular nexopathy paradigm) in FTD (Warren et al., 2013), the present findings supported the view that at the very early disease stage FTD is affecting brain as global system as well. These findings may have important implication on clinical grounds, as tracking desynchronization of the inner fluctuations of the brain might be a helpful prognostic marker to be used in future pharmacological and prevention trials and it could be considered a feasible approach to identify novel targets of intervention.

\section{Acknowledgements}

This work was supported in part by grants from the NIH (R01REB020407, P20GM103472), NSF grant 1539067 and the Wellcome Trust grant (JBR 103838).

\section{Appendix A. Supplementary data}

Supplementary data to this article can be found online at https://doi. org/10.1016/j.neuroimage.2019.01.080.

Meta-state dynamics through time (panel A), meta-state numbers (panel B), meta-state change points (panel C), and meta-state total distance (panel D) in a representative mutation non carrier (left column) and representative mutation carrier (right column).

The colorbar represents the strength of probability to be in each metastate. X-axis: the six connectivity patterns (Cps) are reported, from 1 to 6 ; Y-axis: time (seconds, after time course discretization in quartiles).

\section{References}

Abrol, A., Chaze, C., Damaraju, E., Calhoun, V.D., 2016. The chronnectome: evaluating replicability of dynamic connectivity patterns in 7500 resting fMRI datasets. Conf Proc IEEE Eng Med Biol Soc 5571-5574, 2016.

Abrol, A., Damaraju, E., Miller, R.L., SDtephen, J.M., Claus, E.D., Mayer, A.R., Calhoun, V.D., 2017. Replicability of time-varying connectivity patterns in large resting state fMRI samples. Neuroimage 163, 160-176.

Allen, E.A., Damaraju, E., Plis, S.M., Erhardt, E.B., Eichele, T., Calhoun, V.D., 2014. Tracking whole-brain connectivity dynamics in the resting state. Cerebr. Cortex 24, 663-676.

Arieli, A., Sterkin, A., Grinvald, A., Aertsen, A., 1996. Dynamics of ongoing activity: explanation of the large variability in evoked cortical responses. Science 273 , 1868-1871.

Avena-Koenigsberger, A., Misic, B., Sporns, O., 2017. Communication dynamics in complex brain networks. Nat. Rev. Neurosci. 19, 17-33.

Bell, A.J., Sejnowski, T.J., 1995. An information-maximization approach to blind separation and blind deconvolution. Neural Comput. 7, 1129-1159.

Benjamini, Y., Hochberg, Y., 1995. Controlling the false discovery rate: a practical and powerful approach to multiple testing. J. Roy. Stat. Soc. B 57 1, 289-300.

Borroni, B., Alberici, A., Premi, E., Archetti, S., Garibotto, V., Agosti, C., Gasparotti, R., Di Luca, M., Perani, D., Padovani, A., 2008a. Brain magnetic resonance imaging structural changes in a pedigree of asymptomatic progranulin mutation carriers. Rejuvenation Res. 11, 585-595.

Borroni, B., Archetti, S., Alberici, A., Agosti, C., Gennarelli, M., Bigni, B., Bonvicini, C., Ferrari, M., Bellelli, G., Galimberti, D., Scarpini, E., Di Lorenzo, D., Caimi, L., Caltagirone, C., Di Luca, M., Padovani, A., 2008b. Progranulin genetic variations in frontotemporal lobar degeneration: evidence for low mutation frequency in an Italian clinical series. Neurogenetics 9, 197-205.

Bowie, C.R., Harvey, P.D., 2006. Administration and interpretation of the trail making test. Nat. Protoc. 1, 2277-2281.

Buckner, R.L., Sepulcre, J., Talukdar, T., Krienen, F.M., Liu, H., Hedden, T., AndrewsHanna, J.R., Sperling, R.A., Johnson, K.A., 2009. Cortical hubs revealed by intrinsic functional connectivity: mapping, assessment of stability, and relation to Alzheimer's disease. J. Neurosci. 29, 1860-1873.

Calhoun, V.D., Adali, T., Pearlson, G.D., Pekar, J.J., 2001. A method for making group inferences from functional MRI data using independent component analysis. Hum. Brain Mapp. 14, 140-151.

Calhoun, V.D., Miller, R., Pearlson, G., Adali, T., 2014. The chronnectome: time-varying connectivity networks as the next frontier in fMRI data discovery. Neuron 84, $262-274$.

Calhoun, V.D., Wager, T.D., Krishnan, A., Rosch, K.S., Seymour, K.E., Nebel, M.B., Mostofsky, S.H., Nyalakanai, P., Kiehl, K., 2017. The impact of T1 versus EPI spatial normalization templates for fMRI data analyses. Hum. Brain Mapp. 38, 5331-5342.

Canolty, R.T., Ganguly, K., Kennerley, S.W., Cadieu, C.F., Koepsell, K., Wallis, J.D., Carmena, J.M., 2010. Oscillatory phase coupling coordinates anatomically dispersed functional cell assemblies. Proc. Natl. Acad. Sci. U. S. A. 107, 17356-17361.

Caroppo, P., Habert, M.O., Durrleman, S., Funkiewiez, A., Perlbarg, V., Hahn, V., Bertin, H., Gaubert, M., Routier, A., Hannequin, D., Deramecourt, V., Pasquier, F., Rivaud-Pechoux, S., Vercelletto, M., Edouart, G., Valabregue, R., Lejeune, P., 
Didic, M., Corvol, J.C., Benali, H., Lehericy, S., Dubois, B., Colliot, O., Brice, A., Le Ber, I., 2015. Lateral temporal lobe: an early imaging marker of the presymptomatic GRN disease? J Alzheimers Dis 47, 751-759.

Chang, C., Glover, G.H., 2010. Time-frequency dynamics of resting-state brain connectivity measured with fMRI. Neuroimage 50, 81-98.

Chen, T., Cai, W., Ryali, S., Supekar, K., Menon, V., 2016. Distinct global brain dynamics and spatiotemporal organization of the salience network. PLoS Biol. 14 e1002469.

Ciric, R., Nomi, J.S., Uddin, L.Q., Satpute, A.B., 2017. Contextual connectivity: a framework for understanding the intrinsic dynamic architecture of large-scale functional brain networks. Sci. Rep. 7, 6537.

Damaraju, E., Allen, E.A., Belger, A., Ford, J.M., McEwen, S., Mathalon, D.H., Mueller, B.A., Pearlson, G.D., Potkin, S.G., Preda, A., Turner, J.A., Vaidya, J.G., van Erp, T.G., Calhoun, V.D., 2014. Dynamic functional connectivity analysis reveals transient states of dysconnectivity in schizophrenia. Neuroimage Clin 5, 298-308.

Damoiseaux, J.S., Rombouts, S.A., Barkhof, F., Scheltens, P., Stam, C.J., Smith, S.M., Beckmann, C.F., 2006. Consistent resting-state networks across healthy subjects. Proc. Natl. Acad. Sci. U. S. A. 103, 13848-13853.

De Luca, M., Beckmann, C.F., De Stefano, N., Matthews, P.M., Smith, S.M., 2006. fMRI resting state networks define distinct modes of long-distance interactions in the human brain. Neuroimage 29, 1359-1367.

de Pasquale, F., Corbetta, M., Betti, V., Della Penna, S., 2018 Oct 15. Cortical cores in network dynamics. Neuroimage 180 (Pt B), 370-382.

Dopper, E.G., Rombouts, S.A., Jiskoot, L.C., den Heijer, T., de Graaf, J.R., de Koning, I., Hammerschlag, A.R., Seelaar, H., Seeley, W.W., Veer, I.M., van Buchem, M.A., Rizzu, P., van Swieten, J.C., 2014. Structural and functional brain connectivity in presymptomatic familial frontotemporal dementia. Neurology 83, e19-26.

Du, Y., Allen, E.A., He, H., Sui, J., Wu, L., Calhoun, V.D., 2016. Artifact removal in the context of group ICA: a comparison of single-subject and group approaches. Hum. Brain Mapp. 37, 1005-1025.

Faghiri, A., SDtephen, J.M., Wang, Y.P., Wilson, T.W., 2018. Changing brain connectivity dynamics: from early childhood to adulthood, 39, 1108-1117.

Fries, P., 2005. A mechanism for cognitive dynamics: neuronal communication through neuronal coherence. Trends Cognit. Sci. 9, 474- 480.

Gorno-Tempini, M.L., Hillis, A.E., Weintraub, S., Kertesz, A., Mendez, M., Cappa, S.F., Ogar, J.M., Rohrer, J.D., Black, S., Boeve, B.F., Manes, F., Dronkers, N.F., Vandenberghe, R., Rascovsky, K., Patterson, K., Miller, B.L., Knopman, D.S., Hodges, J.R., Mesulam, M.M., Grossman, M., 2011. Classification of primary progressive aphasia and its variants. Neurology 76, 1006-1014.

Hillebrand, A., Tewarie, P., van Dellen, E., Yu, M., Carbo, E.W., Douw, L., Gouw, A.A., van Straaten, E.C., Stam, C.J., 2016. Direction of information flow in large-scale restingstate networks is frequency-dependent. Proc. Natl. Acad. Sci. U. S. A. 113, 3867-3872.

Hindriks, R., Adhikari, M.H., Murayama, Y., Ganzetti, M., Mantini, D., Logothetis, N.K., Deco, G., 2016. Can sliding-window correlations reveal dynamic functional connectivity in resting-state fMRI? Neuroimage 127, 242-256.

Hutchison, R.M., Womelsdorf, T., Gati, J.S., Everling, S., Menon, R.S., 2013. Resting-state networks show dynamic functional connectivity in awake humans and anesthetized macaques. Hum. Brain Mapp. 34, 2154-2177.

Jafri, M.J., Pearlson, G.D., Stevens, M., Calhoun, V.D., 2008. A method for functional network connectivity among spatially independent resting-state components in schizophrenia. Neuroimage 39, 1666-1681.

Jenkinson, M., Beckmann, C.F., Behrens, T.E., Woolrich, M.W., Smith, S.M., 2012. FSL. Neuroimage 62, 782-790.

Jia, H., Hu, X., Deshpande, G., 2014. Behavioral relevance of the dynamics of the functional brain connectome. Brain Connect. 4, 741-759.

Lee, S.E., Sias, A.C., Mandelli, M.L., Brown, J.A., Brown, A.B., Khazenzon, A.M., Vidovszky, A.A., Zanto, T.P., Karydas, A.M., Pribadi, M., Dokuru, D., Coppola, G., Geschwind, D.H., Rademakers, R., Gorno-Tempini, M.L., Rosen, H.J., Miller, B.L., Seeley, W.W., 2017. Network degeneration and dysfunction in presymptomatic C9ORF72 expansion carriers. Neuroimage Clin 14, 286-297.

Liu, J., Liao, X., Xia, M., He, Y., 2018. Chronnectome Fingerprinting: Identifying Individuals and Predicting Higher Cognitive Functions Using Dynamic Brain Connectivity Patterns, vol. 39, pp. 902-915.

Marusak, H.A., Calhoun, V.D., Brown, S., Crespo, L.M., Sala-Hamrick, K., Gotlib, I.H., Thomason, M.E., 2017. Dynamic functional connectivity of neurocognitive networks in children. Hum. Brain Mapp. 38, 97-108.

Mikl, M., Marecek, R., Hlustik, P., Pavlicova, M., Drastich, A., Chlebus, P., Brazdil, M., Krupa, P., 2008. Effects of spatial smoothing on fMRI group inferences. Magn. Reson. Imaging 26, 490-503.

Miller, R.L., Yaesoubi, M., Calhoun, V.D., 2014. Higher dimensional analysis shows reduced dynamism of time-varying network connectivity in schizophrenia patients. Conf Proc IEEE Eng Med Biol Soc 3837-3840, 2014.

Miller, R.L., Yaesoubi, M., Turner, J.A., Mathalon, D., Preda, A., Pearlson, G., Adali, T., Calhoun, V.D., 2016. Higher dimensional meta-state analysis reveals reduced resting fMRI connectivity dynamism in schizophrenia patients. PLoS One 11 e0149849.

Moreno, F., Sala-Llonch, R., Barandiaran, M., Sanchez-Valle, R., Estanga, A., BartresFaz, D., Sistiaga, A., Alzualde, A., Fernandez, E., Marti Masso, J.F., Lopez de Munain, A., Indakoetxea, B., 2013. Distinctive age-related temporal cortical thinning in asymptomatic granulin gene mutation carriers. Neurobiol. Aging 34, 1462-1468.

Morris, J.C., Weintraub, S., Chui, H.C., Cummings, J., Decarli, C., Ferris, S., Foster, N.L., Galasko, D., Graff-Radford, N., Peskind, E.R., Beekly, D., Ramos, E.M., Kukull, W.A., 2006. The Uniform data set (UDS): clinical and cognitive variables and descriptive data from alzheimer disease centers. Alzheimers Dis. Assoc. Disord. 20, 210-216.

O'Callaghan, C., Shine, J.M., Lewis, S.J., Andrews-Hanna, J.R., Irish, M., 2015. Shaped by our thoughts-a new task to assess spontaneous cognition and its associated neural correlates in the default network. Brain Cogn. 93, 1-10.
Onton, J., Makeig, S., 2006. Information-based modeling of event-related brain dynamics. Prog. Brain Res. 159, 99-120.

Parkes, L., Fulcher, B., Yucel, M., Fornito, A., 2018. An evaluation of the efficacy, reliability, and sensitivity of motion correction strategies for resting-state functional MRI. Neuroimage 171, 415-436.

Pascual-Marqui, R.D., Michel, C.M., Lehmann, D., 1995. Segmentation of brain electrical activity into microstates: model estimation and validation. IEEE Trans. Biomed. Eng. 42, 658-665.

Pievani, M., Filippini, N., van den Heuvel, M.P., Cappa, S.F., Frisoni, G.B., 2014a. Brain connectivity in neurodegenerative diseases-from phenotype to proteinopathy. Nat. Rev. Neurol. 10, 620-633.

Pievani, M., Paternico, D., Benussi, L., Binetti, G., Orlandini, A., Cobelli, M., Magnaldi, S., Ghidoni, R., Frisoni, G.B., 2014b. Pattern of structural and functional brain abnormalities in asymptomatic granulin mutation carriers. Alzheimers Dement 10, S354-S363 e351.

Power, J.D., Barnes, K.A., Snyder, A.Z., Schlaggar, B.L., Petersen, S.E., 2012. Spurious but systematic correlations in functional connectivity MRI networks arise from subject motion. Neuroimage 59, 2142-2154.

Premi, E., Cauda, F., Costa, T., Diano, M., Gazzina, S., Gualeni, V., Alberici, A., Archetti, S., Magoni, M., Gasparotti, R., Padovani, A., Borroni, B., 2016. Looking for neuroimaging markers in frontotemporal lobar degeneration clinical trials: a multivoxel pattern analysis study in granulin disease. J Alzheimers Dis 51, 249-262.

Premi, E., Cauda, F., Gasparotti, R., Diano, M., Archetti, S., Padovani, A., Borroni, B., 2014a. Multimodal FMRI resting-state functional connectivity in granulin mutations: the case of fronto-parietal dementia. PLoS One 9 e106500.

Premi, E., Formenti, A., Gazzina, S., Archetti, S., Gasparotti, R., Padovani, A., Borroni, B., 2014b. Effect of TMEM106B polymorphism on functional network connectivity in asymptomatic GRN mutation carriers. JAMA Neurol 71, 216-221.

Premi, E., Grassi, M., van Swieten, J., Galimberti, D., Graff, C., Masellis, M., Tartaglia, C., Tagliavini, F., Rowe, J.B., Laforce Jr., R., Finger, E., Frisoni, G.B., de Mendonca, A., Sorbi, S., Gazzina, S., Cosseddu, M., Archetti, S., Gasparotti, R., Manes, M., Alberici, A., Cardoso, M.J., Bocchetta, M., Cash, D.M., Ourselin, S., Padovani, A., Rohrer, J.D., Borroni, B., 2017. Cognitive reserve and TMEM106B genotype modulate brain damage in presymptomatic frontotemporal dementia: a GENFI study. Brain 140, 1784-1791.

Preti, M.G., Bolton, T.A., Van De Ville, D., 2017. The dynamic functional connectome: state-of-the-art and perspectives. Neuroimage 160, 41-54.

Rascovsky, K., Hodges, J.R., Knopman, D., Mendez, M.F., Kramer, J.H., Neuhaus, J., van Swieten, J.C., Seelaar, H., Dopper, E.G., Onyike, C.U., Hillis, A.E., Josephs, K.A., Boeve, B.F., Kertesz, A., Seeley, W.W., Rankin, K.P., Johnson, J.K., GornoTempini, M.L., Rosen, H., Prioleau-Latham, C.E., Lee, A., Kipps, C.M., Lillo, P., Piguet, O., Rohrer, J.D., Rossor, M.N., Warren, J.D., Fox, N.C., Galasko, D., Salmon, D.P., Black, S.E., Mesulam, M., Weintraub, S., Dickerson, B.C., DiehlSchmid, J., Pasquier, F., Deramecourt, V., Lebert, F., Pijnenburg, Y., Chow, T.W., Manes, F., Grafman, J., Cappa, S.F., Freedman, M., Grossman, M., Miller, B.L., 2011. Sensitivity of revised diagnostic criteria for the behavioural variant of frontotemporal dementia. Brain 134, 2456-2477.

Rashid, B., Arbabshirani, M.R., Damaraju, E., Cetin, M.S., Miller, R., Pearlson, G.D., Calhoun, V.D., 2016. Classification of schizophrenia and bipolar patients using static and dynamic resting-state fMRI brain connectivity. Proc. Natl. Acad. Sci. U. S. A. 134, 645-657.

Rashid, B., Damaraju, E., Pearlson, G.D., Calhoun, V.D., 2014. Dynamic connectivity states estimated from resting fMRI Identify differences among Schizophrenia, bipolar disorder, and healthy control subjects. Front. Hum. Neurosci. 8, 897.

Rohrer, J.D., Nicholas, J.M., Cash, D.M., van Swieten, J., Dopper, E., Jiskoot, L., van Minkelen, R., Rombouts, S.A., Cardoso, M.J., Clegg, S., Espak, M., Mead, S., Thomas, D.L., De Vita, E., Masellis, M., Black, S.E., Freedman, M., Keren, R., MacIntosh, B.J., Rogaeva, E., Tang-Wai, D., Tartaglia, M.C., Laforce Jr., R., Tagliavini, F., Tiraboschi, P., Redaelli, V., Prioni, S., Grisoli, M., Borroni, B., Padovani, A., Galimberti, D., Scarpini, E., Arighi, A., Fumagalli, G., Rowe, J.B., CoyleGilchrist, I., Graff, C., Fallstrom, M., Jelic, V., Stahlbom, A.K., Andersson, C., Thonberg, H., Lilius, L., Frisoni, G.B., Pievani, M., Bocchetta, M., Benussi, L., Ghidoni, R., Finger, E., Sorbi, S., Nacmias, B., Lombardi, G., Polito, C., Warren, J.D., Ourselin, S., Fox, N.C., Rossor, M.N., Binetti, G., 2015. Presymptomatic cognitive and neuroanatomical changes in genetic frontotemporal dementia in the Genetic Frontotemporal dementia Initiative (GENFI) study: a cross-sectional analysis. Lancet Neurol. 14, 253-262.

Sakoglu, U., Pearlson, G.D., Kiehl, K.A., Wang, Y.M., Michael, A.M., Calhoun, V.D., 2010. A method for evaluating dynamic functional network connectivity and taskmodulation: application to schizophrenia. Magma 23, 351-366.

Shakil, S., Lee, C.H., Keilholz, S.D., 2016. Evaluation of sliding window correlation performance for characterizing dynamic functional connectivity and brain states. Neuroimage 133, 111-128.

Shine, J.M., Koyejo, O., Bell, P.T., Gorgolewski, K.J., Gilat, M., Poldrack, R.A., 2015 Estimation of dynamic functional connectivity using Multiplication of Temporal Derivatives. Neuroimage 122, 399-407.

Van Dijk, K.R., Sabuncu, M.R., Buckner, R.L., 2012. The influence of head motion on intrinsic functional connectivity MRI. Neuroimage 59, 431-438.

Wang, C., Ong, J.L., Patanaik, A., Zhou, J., 2016. Spontaneous Eyelid Closures Link Vigilance Fluctuation with fMRI Dynamic Connectivity States, vol. 113, pp. 9653-9658.

Warren, J.D., Rohrer, J.D., Hardy, J., 2012. Disintegrating brain networks: from syndromes to molecular nexopathies. Neuron 73, 1060-1062.

Warren, J.D., Rohrer, J.D., Schott, J.M., Fox, N.C., Hardy, J., Rossor, M.N., 2013. Molecular nexopathies: a new paradigm of neurodegenerative disease. Trends Neurosci. 36, 561-569. 
Wear, H.J., Wedderburn, C.J., Mioshi, E., Williams-Gray, C.H., Mason, S.L., Barker, R.A., Hodges, J.R., 2008. The Cambridge behavioural inventory revised. Dement Neuropsychol 2, 102-107.

Whitwell, J.L., Jack Jr., C.R., Boeve, B.F., Senjem, M.L., Baker, M., Rademakers, R. Ivnik, R.J., Knopman, D.S., Wszolek, Z.K., Petersen, R.C., Josephs, K.A., 2009. Voxelbased morphometry patterns of atrophy in FTLD with mutations in MAPT or PGRN. Neurology 72, 813-820.

Whitwell, J.L., Josephs, K.A., Avula, R., Tosakulwong, N., Weigand, S.D., Senjem, M.L. Vemuri, P., Jones, D.T., Gunter, J.L., Baker, M., Wszolek, Z.K., Knopman, D.S.,

Rademakers, R., Petersen, R.C., Boeve, B.F., Jack Jr., C.R., 2011. Altered functional connectivity in asymptomatic MAPT subjects: a comparison to bvFTD. Neurology 77 866-874
Yaesoubi, M., Allen, E.A., Miller, R.L., Calhoun, V.D., 2015. Dynamic coherence analysis of resting fMRI data to jointly capture state-based phase, frequency, and time-domain information. Neuroimage 120, 133-142.

Yan, C.G., Wang, X.D., Zuo, X.N., Zang, Y.F., 2016. DPABI: data processing \& analysis for (Resting-State) brain imaging. Neuroinformatics 14, 339-351.

Yanagawa, T., Mogi, K., 2009. Analysis of ongoing dynamics in neural networks. Neurosci. Res. 64, 177-184.

Zhou, J., Greicius, M.D., Gennatas, E.D., Growdon, M.E., Jang, J.Y., Rabinovici, G.D., Kramer, J.H., Weiner, M., Miller, B.L., Seeley, W.W., 2010. Divergent network connectivity changes in behavioural variant frontotemporal dementia and Alzheimer's disease. Brain 133, 1352-1367. 